Proyecciones

Vol. $11 \mathrm{~N}^{\circ} 1$, pp.45-62 Mayo 1992

Universidad Católica del Norts

Autolagasta - Chile

\title{
EXISTENCIA Y COMPORTAMIENTO ASINTOTICO DE UNA CLASE DE ECUACIONES CON RETARDO
}

\author{
JULIO GALLARDO* \\ Universidad de La Frontera, Teruuco. Chile \\ MANUEL PINTO** \\ Iniversidad de Chile, Santiago, Chile.
}

By means of Tychonoff 's Theorem on the fixed poind, we abtain exastence thenr:ms and asymplotic formulat for the solutions of a certain class of delay-differental equations

* Research supported by Facultad de Ciencias and Proyectos D.I. 120-2-60 Universidad de La Sererra

** Research supported by grants [).T.I. Universidad de C'hile E 3063-9222. and Fondecy $10855-91$ 


\section{Introducción.}

Sean $\tau$ y $b$ dos números reales positivos, $I=[0, \infty)$ y $B_{n}[0, b] \subseteq \mathbf{R}^{n}$ la bola cerrada centrada en el origen y de radio $b$. Sea $B: l-M_{n}(R)$ una función matricial. En lo sucesivo, $|\cdot|$ representa una norma espectral de matrices. Definamos $\dot{B}(t)=B(t)$ si $t \geq 0, \dot{B}(t)=0$ si $t<0$ y $\left\|B_{t}\right\|=\sup _{t-\tau \leq s \leq t}|\hat{B}(s)|, \quad\left\|B_{t}\right\|_{2}=\sup _{t-2 \tau \leq s \leq t}|B(s)|$ para $t \geq 0 y$ $\|B\|_{\infty}=\sup _{s \geq 0}|B(\bar{s})|$.

Sea $r: I \times B_{n}[0, b] \rightarrow[0, \tau]$ una función continua y considere para $t \geq 0$ :

$$
m_{\rho}(t)=\sup _{|\Phi-1(t) z| \leq 1} r(t, z) . \quad \rho>0
$$

donde $\Phi$ es una matriz fundamental del sistema

$$
\dot{y}=A(t) y \text {. }
$$

Para $\Phi$ acotada, nosotros denotamos $m_{b}=m_{e^{\prime \prime}}$, donde $\varrho_{n}=b /\|\Phi\|_{\infty}$

Finalmente, se definen las funciones:

$$
\lambda_{b}(t)=|A(t)|\left\|A_{t}\right\| \cdot\left|\Phi^{-1}(t)\right|\left\|\Phi_{t}\right\|_{2} \cdot m_{b}(t)
$$

$y$

$$
\lambda_{\rho}(t)=|A(t)|\left\|A_{t}\right\| \cdot\left|\Phi^{-1}(t)\right|\left\|\Phi_{t}\right\|_{2} \cdot m_{\rho}(t)
$$

Hemos probado el siguiente teorema.

Teorema 1. Supongamos que $\Phi$ es acotada, que $\lambda_{6} \in L_{1}(I)$ y sea $0<\rho \leq$ $\frac{b}{\|\Phi\|_{\infty}}$. Entonces

i) Para cada $\xi \in \mathbf{R}^{n} .|\xi|<\rho$ y $t_{0} \geq 0$ que satisfagan $|\xi|+3 \rho \int_{t_{0}}^{\infty} \lambda_{b}(s) d s \leq \rho$, existe una solución $x$ de la ecuación

$$
\dot{x}(t)=A(t) x(t-r(t, x(t)))
$$

definida para $t \geq t_{0}$ que satisface $\left|\Phi^{-1}(t) x(t)\right| \leq \rho$ y 


$$
x(t)=\Phi(t)\left[\xi+0\left(\int_{t}^{\infty} \lambda_{b}(s) d s\right)\right], \quad t \rightarrow \infty
$$

ii) Además, dada cualquier solución $x$ de (1) definida para $t \geq s_{0}$, que satisfaga $\left|\Phi^{-1}(t) x(t)\right| \leq \rho$, para $t \geq s_{0}$; existe $\xi \in \mathbf{R}^{n},|\xi| \leq \rho$. tal que se verifica (2).

Este resultado mejora el obtenido por Cooke en [3], quien establecio:

Teorema 2. Sea $r(u)$ definida, no negativa y continua para $|u| \leq b$ y supongamos que las siguientes condiciones son satisfechas:

$$
r(0)=0, \quad \int_{t_{0}}^{\infty} m(t) d t<\infty
$$

donde

$$
m(t)=\sup _{|u| \leq b e^{-a t}} r(u)
$$

Entonces, existe $c^{\prime}>0$ con la propiedad siguiente: Si $r$ esta en $\left[-c^{\prime}, c^{\prime}\right]$, hay una solución $u(t)$ de la ecuación

$$
\dot{u}(t)=-a u(t-r(u(t))), \quad a>0
$$

definida en una vecindad de $+\infty$, que satisface

$$
\lim _{t \rightarrow+\infty} u(t) e^{a t}=c
$$

Recíprocamente, suponiendo condiciones bajo las cuales la solución de ${ }^{*}$ ) esta unicamente determinada por una función inicial, entonces cada solución de $\left({ }^{*}\right)$ definida en una vecindad de $+\infty$ y que sea suficientemente pequeña sobre un intervalo inicial adecuado, satisface $\left({ }^{* *}\right)$ para alguna constante $c$.

Las mejoras son evidentes:

1. La ecuación (1) es vectorial y $\left(^{*}\right)$ es escalar

2. Se cambio la constante $a>0$ por $A(t)$ matriz continua 
3. Se cambio un $r[-b,+b] \rightarrow[0, \tau]$ por $r \quad I \times B_{n}[0, b]-[0, \tau]$

4. En nuestro resultado, para cada $|\xi|<b$. existe solución $x$ de (1) qur. satisface (2)

Cooke solo puede asegurar que para cada $c$ en un intervalo contenido en $\left(-\frac{b}{4},+\frac{b}{4}\right)$, existe solución $x$ de $\left(^{*}\right)$ que satisface $\left(^{* *}\right)$.

5. En nuestro resultado, cualquier solución $x$ de (1) que verifica $\left|\Phi^{-1}(t) x(\ell)\right|$ $\leq \rho$, satisface la fórmula asintótica (2). Cooke necesita que $x$ sea suficientemente chica en cierto intervalo inicial, para que se verifique $\left(^{* *}\right)$.

En $[4,8-10]$ se han obtenido fórnulas asintóticas para retardos dependientes sólo del tiempo. En [II], la ecuación estudiada no es asintóticamente parecida a una ecuación ordinaria. En [7], se insinua por primera vez la clase de funciones de Lipschitz (' usada en la demostración de la parte i) del Teorema 1. En [5. 7] se' estudian ecuaciones con retardos dependientes tanto del tiempo cono del estado.

\section{Demostración del Teorema 1 .}

Parte ii) Sea $x$ una solución de (1), definida sobre $\left[s_{0},+\infty\right)$. Entonces

$$
y(t)=x(t)-\Phi(t) \int_{t_{0}}^{t} \Phi^{-i}(s) A(s)[x(s-r(s, x(s))-x(s)] d s
$$

donde $t_{0} \geq s_{0}+\tau$, es una solución le $y=A(t) y$

En efecto:

$$
\begin{aligned}
\dot{y}(t) & =\dot{x}(t)-\Phi(t) \Phi^{-1}(t) A(t)[x(t-r(t, x(t))-x(t)] \\
& -A(t) \Phi(t) \int_{t_{0}}^{t} \Phi^{-1}(s) A(s)[x(s-r(s, x(s))-x(s)] d s \\
& =A(t) x(t-r(t, x(t)))-A(t) x(t-r(t, x(t)))+A(t) x(t) \\
& -A(t) \Phi(t) \int_{t_{0}}^{t} \Phi^{-1}(s) A(s)[x(s-r(s, x(s)))-x(s)] d s \\
& =A(t) y(t) .
\end{aligned}
$$

Por lo tanto, existe $c \in \mathbf{R}^{n}$ tal que $y(t)=\Phi(t) c$ y entonces 


$$
r(t)=\Phi(t)\left[c+\int_{i_{\mathrm{n}}}^{t} \Phi^{-1}(s) A(s)[r(s-r(s, x(s)))-x(s)] d s\right]
$$

Ademàs

$$
\left|\Phi^{-1}(s) A(s)[x(s-r(s, x(s)))-x(s)]\right| \leq \rho \lambda_{4}(s)
$$

En efecto: Dados $t, t^{\prime} \geq s_{0}+\tau, 0 \leq t-t^{\prime} \leq \tau$, cono $x$ es clerivable sobre $\left[s_{0},+\infty\right)$, se tiene:

$$
x(t)-s\left(t^{\prime}\right)=\int_{t^{\prime}}^{t} x(s) d s
$$

Pero

$$
\begin{aligned}
|\dot{x}(s)| & =|A(s) x(s-r(s, \alpha(s)))| \leq|A(s)||x(s-r(s, s(s)))| \\
& =|A(s)|\left|\boldsymbol{D}(s-r(s, t(s))) \Phi^{-1}(s-r(s, x(s))) x(s-r(s, x(s)))\right| \\
& \leq \rho|A(s)||\Phi(s-r(s, r(s)))| .
\end{aligned}
$$

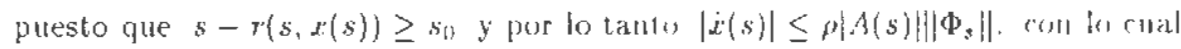

$$
\left|x(t)-x\left(t^{\prime}\right)\right| \leq \int_{t^{\prime}}^{t}|\dot{x}(s)| d s \leq \int_{t^{\prime}}^{t} \rho|A(s)| \| \Phi_{s}|| d s
$$

$y$ como $0 \leq t-t^{\prime} \leq T$, entonces

$$
|A(s)| \leq\left\|A_{i}\right\| \quad y \quad\left\|\Phi_{s}\right\| \leq\left\|\Phi_{t}\right\|_{2}
$$

De donde:

$$
\left.\mid x(t)-s\left(t^{\prime}\right)\right] \leq p\left\|A _ { t } \left|\left\||| \Phi_{t}\right\|_{2}\left(t-t^{\prime}\right)\right.\right.
$$

para cualquier $t, t^{\prime} \geq s_{0}+\tau \quad y \quad 0 \leq t-t^{\prime} \leq \tau$.

Así, para $s \geq s_{0}+2 \tau$. se tiene que $s-r(s, x(s)) \geq s_{0}+\tau$, por lo tanto

$$
\begin{aligned}
\left|\Phi^{-1}(s) A(s)[x(s-r(s, x(s)])-x(s)]\right| & \leq\left|\Phi^{-1}(s)\right|\left\|_{i}(s)|\rho| \mid A(s)\right\|\left\|\Phi_{s}\right\|_{2} r(s, x(s)] \\
& \leq \rho \mid A(s)\left\|A_{s}\right\|\left\|\Phi^{-1}(s)\right\|\left\|\Phi_{s}\right\|_{2} m_{b}(s) \\
& =\rho \lambda_{b}(s)
\end{aligned}
$$


Con esto, podemos escribir:

$$
\begin{aligned}
x(t)= & \Phi(t)\left[c+\int_{t_{0}}^{\infty} \Phi^{-1}(s) A(s)[x(s, r(s, x(s)))-s(s)] d s\right. \\
& \left.-\int_{t}^{\infty} \Phi^{-1}(s) A(s)[x(s-r(s, x(s)))-x(s)] d s\right]
\end{aligned}
$$

Es decir

$$
x(t)=\Phi(t)\left[\xi+0\left(\int_{1}^{\infty} \lambda_{b}(s) d s\right)\right], \quad t \rightarrow \infty
$$

donde

$$
\xi=c+\int_{t_{0}}^{\infty} \phi^{-1}(s) A(s)[x(s-r(s x(s)))-x(s)] d s
$$

que necesariamente verifica $|\xi| \leq \rho$, ya que $\left|\Phi^{-J}(t) x(t)\right| \leq \rho_{1}$ para $t \geq s_{t}$.

Parte i) Sea $E=c\left(\left[t_{0}-\tau,+\infty\right), \mathbf{R}^{n}\right)$ el espacio vectorial topuleigicn ido las funciones continuas en $\left[l_{u}-\tau,+\infty\right)$, dotado de la topología corrpactuabierta. Sea $C \subseteq E$ definido por $x \in C$ si y sólo si $x$ es constand. sobro $\left[\iota_{0}-\tau, \iota_{0}\right],\left|\Phi^{-1}(t) x(t)\right| \leq \rho$, para $t \geq t_{0}$ y $\left|x(t)-x\left(t^{\prime}\right)\right| \leq 3 \rho\left\|A_{t} \mid\right\| \Phi_{t} \|_{2}\left(t-t^{\prime}\right)$ para $t, t^{\prime} \geq t_{0}, 0 \leq t-t^{\prime} \leq \tau$.

Definamos la función

$$
\begin{aligned}
T: C^{\prime} & \rightarrow E \\
x & \rightarrow T(x)=y
\end{aligned}
$$

dada por

$$
\begin{gathered}
y(l)=\Phi(t)\left[\xi-\int_{t}^{\infty} \Phi^{-1}(s) A(s)[x(s-r(s, x(s)))-x(s)] d s\right], t \geq t_{0} \\
y(t)=y\left(t_{0}\right) ; \quad t_{0}-\tau \leq t \leq t_{0}
\end{gathered}
$$

$T$ esta biem definida pues:

(3)

$$
\left|\Phi^{-1}(s) A(s)[x(s-r(s, x(s)))-x(s)]\right| \leq 3 \rho \lambda_{b}(s)
$$

$y$ claramente $y \in E$.

Debemos probar que $T$ verifica: 
1. $T(C) \subseteq C$

2. $T$ es continua solire $C$, en la topología compacta abierta.

3. $C$ es cerrado, convexo y compacto.

Usando entonces el tcorouch del punto fijo de Tychonotf [6], pudernos asisgurar que existe $x \in C:$ tal que $T(x)=x$, es decir:

$$
\begin{aligned}
& x(t)=\Phi(t)\left[\xi-\int_{t}^{\infty} \Phi^{-1}(s) A(s)[x(s-r(s, x(s)))-x(s)] d(s], t \geq t_{0}\right) \\
& x(t)=x\left(t_{0}\right), \quad t_{0}-\tau \leq t \leq t_{0} .
\end{aligned}
$$

Derivando, se obliene:

$$
x(t)=A(t) x(t-r(t, x(t))) \text { para todo } t \geq \ell_{i}
$$

y tenjendo en cuenta (3), se tiene:

$$
x(t)=\Phi(t)\left[\xi+0\left(\int_{t}^{\infty} \lambda_{b}(s) d s\right)\right], \quad t-\infty
$$

Denostración de (1). Dado $x \in C^{\prime}$, sea $y=T^{\top}(x)$.

Es obvjo, por la definición de $T$. que $y$ as constante sobre $\left[t_{11}-T \cdot t_{01}\right]$ Por otea parte:

$$
\begin{aligned}
\left|\Phi^{-1}(t) y(t)\right| & \leq|\xi|+\int_{t}^{\infty}\left|\Phi^{-1}(s)\|A(s)\| x(s-r(s, x(s)))-x(s)\right| d s \\
& \leq|\xi|+\int_{t}^{\infty}\left|\Phi^{-1}(s)\|A(s) \mid 3 \rho\| A_{s}\|\| \Phi_{s} \|_{2} \cdot m_{b}(s) d s\right. \\
& \leq|\xi|+3 \rho \int_{t_{0}}^{\infty} \lambda_{b}(s) d s \leq \rho \text { para todo } t \geq t_{0}
\end{aligned}
$$

Además, para $s \geq t_{0}$.

$$
\dot{y}(s)=A(s) y(s)+A(s)[x(s-r(s, x(s)))-x(s)]
$$


Si suponemos $s-r(s, x(s)) \geq t_{0}$, entonces, como

$$
\left|\Phi^{-1}(s) y(s)\right| \leq \rho,\left|\Phi^{-1}(s) x(s)\right| \leq \rho \quad \text { para } \quad s \geq t_{0}
$$

se tiene:

$$
\left|\Phi^{-1}(s-r(s, x(s))) x(s-r(s, x(s)))\right| \leq \rho,
$$

entonces:

$$
\begin{aligned}
|\dot{y}(s)| & \leq|A(s)||y(s)|+|A(s)||x(s-r(s, x(s)))|+|A(s)||x(s)| \\
& =|A(s)|\left|\Phi(s) \Phi^{-1}(s) y(s)\right| \\
& +|A(s)|\left|\Phi(s-r(s, x(s))) \Phi^{-1}(s-r(s, x(s))) x(s-r(s, x(s)))\right| \\
& +|A(s)|\left|\Phi(s) \Phi^{-1}(s) x(s)\right| \\
& \leq \rho|A(s)\|\Phi(s)|+\rho| A(s)|| \Phi(s-r(s, x(s)))|+\rho| A(s)\| \Phi(s)| \\
& \leq 2 \rho|A(s)||\Phi(s)|+\rho|A(s)|\|\Phi,\| .
\end{aligned}
$$

Si suponemos $s-r(s, x(s))) \leq t_{0}$, entonces, como $x(s-r(s, x(s)))=x\left(t_{0}\right)$ se tendra $\left|\Phi^{-1}\left(t_{0}\right) x\left(t_{0}\right)\right| \leq \rho$. Por lo tanto:

$$
\begin{aligned}
|\dot{y}(s)| & \leq\left|A(s)\left\|\Phi(s) \Phi^{-1}(s) y(s)|+| A(s)\right\| \Phi\left(t_{0}\right) \Phi^{-1}\left(t_{0}\right) x\left(t_{0}\right)\right| \\
& +|A(s)|\left|\Phi(s) \Phi^{-1}(s) x(s)\right| \\
& \leq \rho|A(s)||\Phi(s)|+\rho\left|A(s) \| \Phi\left(t_{0}\right)\right|+\rho|A(s)||\Phi(s)| \\
& \leq 2 \rho\left|A(s)\|\Phi(s)|+\rho| A(s) \mid\| \Phi_{s} \| .\right.
\end{aligned}
$$

Luego, para $t, t^{\prime} \geq t_{0}, 0 \leq t-t^{\prime} \leq \tau$, se tiene:

$$
\begin{aligned}
\left|y(t)-y\left(t^{\prime}\right)\right| & \leq \int_{t^{\prime}}^{t}|y(s)| d s \leq \int_{t^{\prime}}^{t}\left(2 \rho\left|A(s)\|\| \Phi_{s}\|+\rho|A(s)| \cdot\| \Phi_{s} \|\right) d s\right. \\
& \leq \int_{t^{\prime}}^{t} 3 \rho\left\|A_{t}\right\| \cdot\left\|\Phi_{t}\right\|_{2} d s=3 \rho\left\|A_{t}\right\| \cdot\left\|\Phi_{t}\right\|_{2}\left(t-t^{\prime}\right),
\end{aligned}
$$

ya que $0 \leq t-t^{\prime} \leq \tau$ y $t^{\prime} \leq s \leq t$ implica

$$
|A(s)| \leq\left\|A_{t}\right\| \text { y }|\Phi(s)| \leq\left\|\Phi_{s}\right\| \leq\left\|\Phi_{t}\right\|_{2} .
$$


Así hemos probado que $T(C) \subseteq C$

Demostración de (2). Sea $\left\{x_{n}\right\}_{n}$ una sucesión en $C^{*}$ y $x$ en $C$. tal que $x_{n} \rightarrow x$ en la topología compacta abierta.

Debemos probar que $T\left(x_{n}\right) \rightarrow T(x)$.

En primer lugar, veamos que para cualquier $s \geq t_{0}$

$$
\lim _{n \rightarrow \infty} \Phi^{-1}(s) A(s)\left[\left(x(s-r(s, x(s)))-x_{n}\left(s-r\left(s, x_{n}(s)\right)\right)\right)+\left(x_{n}(s)-x(s)\right)\right]=0
$$

Para abreviar escribamos

$$
\Delta_{n}(x, s)=A(s)\left[\left(x(s-r(s, x(s)))-x_{n}\left(s-r\left(s, x_{n}(s)\right)\right)\right)+\left(x_{n}(s)-x(s)\right)\right]
$$

y sea $t_{0} \leq s \leq L$. con $L$ arbitrario fijo.

Con esto

$$
\left|\Phi^{-1}(s) \Delta_{n}(x . s)\right| \leq M\left|\left(x(s-r(s, x(s)))-x_{n}\left(s-r\left(s, x_{n}(s)\right)\right)\right)+\left(x_{n}(s)-x(s)\right)\right|
$$

para todo $s \in\left[t_{0} . L\right]$. para cierta constante $M$

Además, por la continuidad uniforme de $x$ sobre $\left[t_{0}-\tau, L\right]$, la de $r$ sobre $\left[t_{0}, L\right] \times B_{n}[0, b]$ y la convergencia uniforme de $x_{n}$ hacia $x$ sobre $\left[t_{0}-\tau, L\right]$, tenemos que existe $\delta>0$ tal que $\left|t-t^{\prime}\right|<\delta ; t, t^{\prime} \in\left[t_{0}-\tau, L\right]$ implican $\left|x(t)-x\left(t^{\prime}\right)\right|<\varepsilon / M$ Además, existe $\delta^{\prime}>0$ tal que

$$
(s, x),\left(s, x^{\prime}\right) \in\left[t_{0}, L\right] \times B_{n}[0, b] ;\left|(s, x)-\left(s, x^{\prime}\right)\right|<\delta^{\prime}
$$

implica $\left|r(s, x)-r\left(s, x^{\prime}\right)\right|<\delta$ y finalmente existe $N$ tal que $n \geq N$ implica

$$
\left|x_{n}(s)-x(s)\right|<\frac{\varepsilon}{3 M}
$$

y

$$
\left|x_{n}(s)-x(s)\right|<\delta^{\prime} . \text { para } s \in\left[t_{0}-\tau, L\right] .
$$


Por lo tanto, para $\left.n \geq N, \mid(s, n(s))-\left(s, n_{n 2}(s)\right)\right\} \mid<\delta^{\prime}$ y como

$$
(s, s(s)),\left(s, s_{n}(s)\right) \in\left[l_{0}, l\right] \times B_{n}[0, b]
$$

se tiene

$$
\left|r(s, x(s))-r\left(s, x_{n}(s)\right)\right|<\delta
$$

o equivalentemente

$$
\left.\mid(s-v(s, n(s)))-\left(s-v\left(s, x_{n}\right)\right)\right\} \mid<\delta \text {. }
$$

konde

$$
s-r(s, x(s)), s-r\left(s, x_{n}(s)\right) \in\left[l_{0}-\tau, L\right] .
$$

Esto implica

$$
\left|x(s-r(s, x(s)))-2\left(s-r\left(s, x_{n}(s)\right)\right)\right|<\frac{\varepsilon}{3 M}
$$

Por otro lado, como s $\in\left[\ell_{0}, L\right]$ y $s-r\left(s, x_{n}(s)\right) \in\left[\ell_{0}-\tau, L\right], \quad n \geq N$ implira $\left|x_{n}(s)-x(s)\right|<\frac{z}{3 n} y$

$$
\left|x_{n}\left(s-r\left(s, x_{n}(s)\right)\right)-s\left(s-r\left(s, x_{n}(s)\right)\right)\right|<\frac{c}{3 M}
$$

Todo lo anterior permite que, para $n \geq N$

$$
\begin{aligned}
\left|\Phi^{-1}(s) \Delta_{n}(x, s)\right| & \leq M\left(\left|x(s-v(s, x(s)))-x\left(s-r\left(s, x_{n}(s)\right)\right)\right|\right. \\
& \left.+\left|x\left(s-r\left(s, x_{n}(s)\right)\right)-x_{n}\left(s-r\left(s, x_{n}(s)\right)\right)\right|+\left|x_{n}(s)-x(s)\right|\right) \\
& <M\left(\frac{\varepsilon}{3 M}+\frac{\varepsilon}{3 M}+\frac{\varepsilon}{3 M}\right)=\varepsilon
\end{aligned}
$$

o sea, cualquiera sea $s \geq t_{0}, \lim _{n \rightarrow \infty} \Phi^{-1}(s) \Delta_{n}(2, s)=0$.

Aplicando el teorema de la convergencia dominada the Lebesgue. y a que por (3) $\left|\Phi^{-1}(s) \Delta_{n}\left(2^{2}, s\right)\right| \leq 6 \varrho \lambda_{b}(s)$, tenemos: 


$$
\lim _{n \rightarrow \infty} \int_{t_{0}}^{\infty}\left|\Phi^{-1}(s) \Delta_{n}\left(s^{\prime}, s\right)\right| d s=0
$$

y por lo tanto, dado cualquier compacto $\left[l_{0}, \gamma\right]$.

$$
\lim _{n \rightarrow \infty}|\Phi(t)| \int_{t_{11}}^{\infty}\left|\Phi^{-1}(s) \Delta_{n}(x, s)\right| r l s=0
$$

uniformenente sobre $\left[t_{0}, T\right]$.

Fijalmente:

$$
\begin{aligned}
\left|T\left(x_{n}\right)(t)-T(x)(t)\right| & =\left|d(l) \int_{t}^{\infty} p^{-1}(s) \Delta_{n}(x, s) d s\right| \\
& \leq|d(t)|\left|\int_{t}^{\infty} \phi^{-1}(s) \Delta_{n}(x, s) d s\right| \\
& \leq|d(1)| \int_{t_{0}}^{a}\left|\Phi^{-1}(s) \Delta_{n}(s, s)\right| d s \rightarrow 0
\end{aligned}
$$

uniformenente sobre $\left[t_{0}, T\right]$.

Demostración de (3). $C$ es evidentenente cerado y convexo. Probaremos que $C$ es compacto. Veamos que fijado un compacto $\left[l_{0}, L\right]$, cada $x \in C$ es Lipschitziana sobre $\left[t_{0}, L\right]$, con una misma comstante de Lipschitz y por lo tanto $C$ es equicontinua sobre $\left[t_{u}, L\right]$.

Gil efecto, dados $t, l^{\prime} \in\left[t_{0}, L\right], l \geq l^{\prime}$, existe $n \in \mathbf{N}$ tal que

$$
t^{\prime}+(n-1) \tau<t \quad \text { y } t^{\prime}+u t \geq t
$$

Definanos $t_{t}^{\prime}=t^{\prime}+i T_{\text {, frara }} i=1, \cdots, n-l$.

C'on esto: 


$$
\begin{aligned}
\left|x(t)-x\left(t^{\prime}\right)\right|= & \left|x(t)-x\left(t_{n-1}^{\prime}\right)\right|+\left|x\left(t_{n-1}^{\prime}\right)-x\left(t_{n-2}^{\prime}\right)\right|+\cdots+\left|x\left(t_{1}^{\prime}\right)-x\left(t^{\prime}\right)\right| \\
\leq & 3 \rho\left\|A_{t}\right\|\left(t-t_{n-1}^{\prime}\right)+3 \rho\left\|A_{t_{n-1}^{\prime}}\right\|\left(t_{n-1}^{\prime}-t_{n-2}^{\prime}\right) \\
& +\cdots+3 \rho\left\|A_{t_{1}^{\prime}}\right\|\left(t_{1}^{\prime}-t^{\prime}\right) \\
\leq & 3 \rho \bar{A}\left(t-t_{n-1}^{\prime}\right)+3 \rho \bar{A}\left(t_{n-1}^{\prime}-t_{n-2}^{\prime}\right)+\cdots+3 \rho A\left(t_{1}^{\prime}-t^{\prime}\right) \\
= & 3 \rho \bar{A}\left(t-t^{\prime}\right), \text { donde } \bar{A}=\max _{s \in\left[t_{0}-\tau, L\right]}|\bar{A}(s)|
\end{aligned}
$$

Además, dada $x \in C^{\prime}$

$$
|x(t)|=\left|\Phi(t) \Phi^{-1}(t) x(t)\right| \leq \rho|\Phi(t)| \leq \rho M a x_{t \in\left[t_{0}, L\right]}|\Phi(t)| .
$$

o sea $C$ es uniformemente acotado sobre $\left[t_{0}, L\right]$.

Por lo tanto, dada cualquier sucesión $\left\{x_{n}\right\}_{n}$ en $C^{\prime}$, podemos aplicar el teorena de Arzela-Ascoli sobre $\left[t_{0}, t_{0}+1\right]$, para obtener una subsucesión que converga uniformemente a cierta función sobre $\left[t_{0}, t_{0}+1\right]$. En seguida, a csta subsucesión obtenida, se le aplica Arzela-Ascoli, para obtener una subsucesión, que converga uniformemente a cierta función sobre $\left[t_{0}, t_{0}+2\right]$. Continuando con este procedimiento, obtenemos una subsucesión de $\left\{x_{n}\right\}_{n}$ que converge a una funcón $x$, uniformemente sobre compactos. Por $(3), x$ pertenece a $C$. Esto prueba que $C$ es secuencialnente compacto, o equivalentemente, compacto.

Esto termina la demostración.

En el caso $b=\infty$, no es necesario que $\Phi$ sea acotada y se establece el siguiente corolario.

Corolario 1. Supongamos que para $\rho>0, \lambda_{\rho} \in L_{1}(I)$.

i) Entonces, para cada $\xi \in \mathbf{R}^{n},|\xi|<\rho$ y $t_{0} \geq 0$ que satisfagan $|\xi|+3 \rho \int_{t_{0}}^{\infty} \lambda_{\rho}(s) d s \leq \rho$, existe una solución $x$ de (1) definicla para $t \geq t_{0}$, que satisface $\left|\Phi^{-1}(t) x(t)\right| \leq \rho$ para $t \geq t_{0}$ y la formula asintótica

$$
x(t)=\Phi(t)\left[\xi+0\left(\int_{t}^{\infty} \lambda_{\rho}(s) d s\right)\right], \quad t \rightarrow \infty .
$$


ii) Para cualepuer solución 2 de (1) definida para $t \geq s_{0}$, que satialiuga $\left|\Phi^{-1}(t) x(t)\right| \leq \rho$ para $t \geq s_{1,}$, existe $\xi \in \mathbf{R}^{n},|\xi| \leq p$ tal que are verifica (4).

\section{Ejemplos}

1. Sea $\gamma(t)=|A(t)| \cdot\left\|A_{t}\right\| \cdot\left|\Phi^{-1}(t)\right|^{\prime} \cdot\left\|\Phi_{t}\right\|_{2}$ y supongannus $\gamma \in L_{1}(l)$.

Entonces, el Teorenal es cierto cualduiera sea el comportaniento acotado del retardo $r=r(l, x)$.

Asi, considerenus la ecuración

$$
x(t)=\frac{1}{t+1} x(t-r(t, x(t)))
$$

donde $r: / \times \mathbf{R} \rightarrow[0, \tau]$ es cualquierit.

So liene $\Phi(t)=1+1, \Phi^{-1}(t)=\frac{1}{i+1},\left\|\Phi_{t}\right\|_{2}=t+1, y$

$$
\left\|A_{t}\right\|=\left\{\begin{array}{rll}
\frac{1}{t-\tau+1} & \text { si } & t \geq \tau \\
1 & \text { si } & 0 \leq t \leq \tau .
\end{array}\right.
$$

Por lo tation

$$
\begin{aligned}
\gamma(l) & =\left|A(l)\left\|A_{t}\right\|\right| \Phi^{-1}(l)\|\| \Phi_{l} \|_{2} \\
& =\frac{1}{l+1} \cdot \frac{1}{l-\tau+1} \cdot \frac{1}{t+1}(t+1)=\frac{1}{(t+1)(t-\tau+1)}
\end{aligned}
$$

si $l \geq T, y$ daratnente $\gamma \in L_{1}(I)$

Aplicando el comario: Cono $\lambda_{n} \in L_{1}(l)$, para todio $\beta>0$,

i) Dado cualquier $\xi$ an $\mathbf{R}$ sed $\rho>0$ tal que $|\xi|<\rho$ y $l_{0} \geq 0$, de manera que $|\xi|+3 \rho \int_{t_{0}}^{\infty} \lambda_{\mu}(s) d s \leq \rho$. Entonces exjste solución $x$ de (5) definida para $t \geq$ to que verifica $|x(t)| \leq \rho(t+1)$ y 


$$
x(t)=(t+1)\left(\xi+0\left(\int_{t}^{\infty} \lambda_{\rho}(s) d s\right)\right), \quad t \rightarrow \infty
$$

Notemos que por (6) $\left|\lambda_{\rho}(t)\right| \leq M \frac{1}{t^{2}}$, para cierta constante $M$.

Esto nos permite escribir:

$$
x(t)=(t+1)\left(\xi+0\left(\frac{1}{t}\right)\right), \quad t \rightarrow \infty
$$

ii) Cualquier solución $x$ de (5) definida para $t \geq s_{0}$, que satisfaga $|x(t)| \leq \rho(t+1)$ para $t \geq s_{0}$, tiene la siguiente expresión asintótica (7) para algún $|\xi| \leq \rho$.

2. Dada la ecuación $\dot{x}(t)=A(t) x(t-r(t))$, donde $r: I \rightarrow[0, \tau]$, suponganos que $\gamma(t) \cdot r(t)$, (donde $\gamma(t)$ fue definicla en ejemplo l) está en $L_{1}(I)$. Entonces, el corolario es aplicable.

Veamos la siguiente ecuación:

$$
\dot{x}(t)=\operatorname{tx}\left(t-\frac{1}{(t+1)^{\alpha}}\right), \quad \text { con } \alpha>3
$$

Aqui $A(t)=t, \Phi(t)=e^{\frac{t^{2}}{2}},\left\|\Phi_{t}\right\|_{2}=e^{\frac{t^{2}}{2}} \quad\left\|A_{t}\right\|=t$ y $\left|\Phi^{-1}(t)\right|=e^{-\frac{t^{2}}{2}}$.

Por lo tanto, $\lambda_{\rho}(t)=t \cdot t \cdot e^{\frac{-t^{2}}{2}} \cdot e^{\frac{t^{2}}{2}} \cdot \frac{1}{(t+1)^{\alpha}}=\frac{t^{2}}{(t+1)^{\alpha}}$.

Luego, aplicando corolario, parte (i):

Para cualquier $\xi \in \mathbf{R}$, sea $\rho>0$ con $|\xi|<\rho$. Escogemos $t_{0} \geq 0$ suficientemente grande, de modo que $|\xi|+3 \rho \int_{t_{0}}^{\infty} \lambda_{\rho}(s) d s \leq \rho$. Entonces, existe solución $x$ de (7), definida para $t \geq t_{0}$, que satisface $|x(t)| \leq \rho e^{\frac{t^{2}}{2}}$, para $t \geq t_{0} \quad y$

$$
x(t)=e^{\frac{t^{2}}{2}}\left[\xi+O\left(\int_{t}^{\infty} \lambda_{\rho}(s) d s\right)\right], \quad t \rightarrow \infty
$$


y de la parte (ii): Toda solución $x$ de (7) definida para $l \geq s_{0}$, que satisfaga $|x(t)| \leq \rho e^{\frac{t^{2}}{2}}$ para $t \geq s_{0}$, para algún $\rho>0$, satisface (8), para algún $\xi \in \mathbf{R},|\xi| \leq \rho$.

3. Consideremos ahora la ecuación (1), suponiendo $r(t, z) \leq K r_{1}(t)$ para $t \geq 0$, donde $K$ es alguna constante positiva y supongamos $\gamma \cdot r_{1} \in L_{1}(I)$.

Entonces nuestros resultados se aplican.

Veamos la ecuación

$$
\dot{x}(t)=x\left(t-\frac{1}{\left(1+t x(t)^{2}\right)(t+1)^{2}}\right)
$$

Aquí $A(t)=1$ y $r: I \times \mathbf{R} \rightarrow[0,1]$.

$$
r(t, z)=\frac{1}{\left(1+t x(t)^{2}\right)(t+1)^{2}}
$$

Se cumple $r(t, z) \leq r_{1}(l)=\frac{1}{(t+1)^{2}} y$

$$
\gamma(t) r_{1}(t)=\left|A(t)\|\| A_{t}\left\|\mid \Phi^{-1}(t)\right\|\left\|\Phi_{t}\right\|_{2} \cdot \frac{1}{(t+1)^{2}}=\frac{1}{(t+1)^{2}}\right.
$$

esta en $L_{1}(I)$.

Por corolario, (ii): Para cualquier solución $x$ de (10) definida para $t \geq s_{0} \geq 0$ que satisfaga $|x(t)| \leq \rho e^{t}$ para $t \geq s_{0}$, existe $\xi$ en $\mathbf{R},|\xi| \leq \rho$ tal que

$$
x(t)=e^{t}\left[\xi+0\left(\int_{t}^{\infty} \frac{d s}{(1+s)^{2}}\right)\right], \quad t \rightarrow \infty
$$

o bien

$$
x(t)=e^{t}\left[\xi+0\left(\frac{1}{1+t}\right)\right], \quad t \rightarrow \infty
$$


Por (i): Dado cualquier $\xi$ en $\mathbf{R}$ y $\rho>0$ tal que $|\xi|<\rho$, escogiendo $\iota_{0} \geq 0$ de manera que $|\xi|+3 \rho \int_{t_{0}}^{\infty} \lambda_{\rho}(s) d s \leq \rho$, existe $x$ solución de (10) definida sobre $\left[t_{0},+\infty\right)$ que satisface $|x(t)| \leq \rho e^{t}$ y la fórmula (11).

4. Conviene destacar que si la condición $\lambda_{1} \in L_{1}(I)$ no se cumple, no necesariamente cualquier solución $x$ de (1) satisface $x(t)=\Phi(t)[\xi+$ $o(1)], t \rightarrow \infty$ En efecto, basta considerar la ecuación

$$
\dot{x}(t)=-x\left(t-\frac{\pi}{2}\right)
$$

En este caso $A(t)=-1$ y por lo tanto

$$
\Phi(t)=e^{-t} \text { y }\left\|\Phi_{t}\right\|_{2}=e^{-\left(t-2 \cdot \frac{\pi}{2}\right)}=e^{\pi} \cdot e^{-t} \text { si } t \geq \pi
$$

Luego

$$
\lambda_{\rho}(t)=|A(t)| \cdot\left\|A_{t}\right\|\left|\Phi^{-1}(t)\right| \cdot\left\|\Phi_{t}\right\|_{2} \cdot m_{\rho}(t)=e^{\pi} \cdot \frac{\pi}{2} \notin L_{1}(I) .
$$

Por otra parte $x(t)=$ sent es solución de (12) y

$$
\Phi^{-1}(t) x(t)=e^{t} \operatorname{sen} t
$$

no es acotada.

5. Consideremos la ecuación

$$
\dot{x}(t)=A x(t-r(t, x(t))),
$$

donde $A$ es una matriz $n \times n$ contante con valores propios no nulos con parte real cero y simples. Si $m_{i} \in L_{1}(I)$, entonces, nuestros resultados son aplicables.

En efecto, bajo estas condiciones $\Phi(t)$ y $\Phi^{-1}(t)$ son acotados, es decir, existe constante $K$ tal que $|\Phi(t)| \leq K^{\prime}$ y $\left|\Phi^{-1}(t)\right| \leq K$. Luego 


$$
\lambda_{i}(t)=|A(t)|\left\|\left|A_{t}\right|\right\|\left|\Phi_{t} \|_{2}\right| \Phi^{-1}(t) \mid \cdot m_{i}(t)
$$

satisface:

$$
\left|\lambda_{i}(t)\right| \leq|A|^{2} K^{2} \cdot m_{i}(t) .
$$

Por lo tanto $\lambda_{i} \in L^{1}(/)$.

Apliquemos por ejemplo el teorema:

i) Dado $0<\rho \leq \frac{b}{\|\Phi\|_{\infty}}$ y $\xi$ en $\mathbf{R}$ lal que $|\xi|<\rho$, escogemus $t_{01} \geq 0$ de manera que $|\xi|+3 \rho \int_{t_{0}}^{\infty} \lambda_{b}(s) d s \leq \rho$. Entonces existe solución $x$ de (13) definida sobre $\left[t_{0},+\infty\right)$, que verifica

$$
x(t)=e^{t A}\left[\xi+0\left(\int_{t}^{\infty} \lambda_{b}(s) d s\right)\right], \quad l \rightarrow \infty
$$

ii) Para cualquier solución $\alpha$ de (1:3) que satisface $\left|e^{-t A} x(t)\right| \leq \rho$, existe $\xi$ ell $\mathbf{R}^{n},|\xi| \leq \rho$ tal que se verifica (14).

\section{REFERENCIAS}

[1] Cooke, K.: Functional chifferential equations close to differential equations, Bull. Amer. Wath. Soc.72, 285-288, 1966.

[2] Cooke, $K$ : Functional differential equations with asynptotically vanishing lag, Rend. Curc. Mat. Palermo XVI, 39-56, 1967.

[3] Cooke, $K$.: Asymptotic theory for the delay-differential equations $u^{\prime}(t)=$ $-a u(t-r(u(t)))$, J. Math. Anal. Appl. 19, 160-173, 1967.

[4] Donoso, A,; Pinto, M.: Asymptotic behavior of solution of $x^{t}(t)=A(l) x(t-$ - $r(t))$, Pre-print Universify of Chile, 1992.

[5] Gallardo, J.; Pinto, M.: Asymtotic integration of delay-differential systemtes with time and state dependent, sibmitted.

[6] Hale, J.: Functional differential equations, Springer-Verlag, 1977. 
[7] Pinto, M.: Asymptotic integration of the functional differential equations, $y^{\prime}(t)=a(t) y(t-r(t, y))$, J. Math. Anal. Appl, 1993, To appear.

[8] Pinto, M.: Green-Liouville formulac for second order delay differential equations, Submilted,

[9] Pinto, M.: Uniform asymptotic stability of solutions of $y^{\prime}(t)=f(t, y(t-$ $r(t)))$, Submilted,

[10] Pinto, M.: Functional differential equations close to ordinary differential equations, Submitted,

[11] Pinto, M.: Asymptotic behavior of solutions of the functional differential equations $x^{\prime}(t)=a(t) x(r(t))+b x(t), \quad b \in \mathbf{R}$, Proyecciones, Vol 10, 59-76, 1991.

Julio Gallardo P.

Departamento de Matemática

Universidad de La Frontera

Casilla 54-D, Temuco, Chile

Manuel Pinto

Departamento de Matemática

Facultad de Ciencias

Universidad de Chile

Casilla 653, Santiago, Chile 